\title{
Co-exposure to refractory ceramic fibres and asbestos and risk of pleural mesothelioma
}

\author{
Aude Lacourt1,2,3, Mickael Rinaldo ${ }^{1,2,3}$, Céline Gramond ${ }^{1,2,3}$, Stéphane Ducamp ${ }^{4}$, \\ Annabelle Gilg Soit Ig $^{5}$, Marcel Goldberg ${ }^{5}$, Jean Claude Pairon ${ }^{6,7}$ and \\ Patrick Brochard ${ }^{1,2,3}$
}

\begin{abstract}
Affiliations: 'Université Bordeaux, ISPED, Centre INSERM U897-Epidemiologie-Biostatistique Equipe Sante Environnement, Bordeaux, France. ${ }^{2}$ INSERM, ISPED, Centre INSERM U897-Epidemiologie-Biostatistique Equipe Sante Environnement, Bordeaux, France. ${ }^{3}$ Université Bordeaux, ISPED, Equipe Associée en Sante Travail, Bordeaux, France. ${ }^{~ I n s t i t u t ~ d e ~ V e i l l e ~ S a n i t a i r e, ~ D e ́ p a r t e m e n t ~ S a n t e ~ T r a v a i l, ~ E q u i p e ~ A s s o c i e ́ e ~ e n ~ S a n t e ~}$ Travail, Bordeaux, France. ${ }^{5}$ Institut de Veille Sanitaire, Département Sante Travail, Saint Maurice, France. 6INSERM U955, Universite Paris-Est Creteil, Faculte de Medecine, Creteil, France. ${ }^{7}$ Centre Hospitalier Intercommunal, Service de pneumologie et pathologie professionnelle, Creteil, France.
\end{abstract}

Correspondence: Aude Lacourt, ISPED-LSTE-ESSAT, 146 Rue Leo Saignat, 33076 Bordeaux, France.

E-mail: aude.lacourtaisped.u-bordeaux2.fr

ABSTRACT The aim of this study was to investigate the hypothesis of an increased risk of pleural mesothelioma due to co-exposure to asbestos and refractory ceramic fibres (RCF) compared to asbestos exposure alone.

Males were selected from a French case-control study conducted in 1987-1993 and from the French National Mesothelioma Surveillance Program in 1998-2006. Two population controls were frequency matched to each case by year of birth. Complete job histories were collected and occupational asbestos and RCF exposures were assessed using job exposure matrices. The dose-response relationships for asbestos exposure were estimated from an unconditional logistic regression model in subjects exposed to asbestos only (group 1) and subjects exposed to both asbestos and RCF (group 2).

A total of 988 cases and 1125 controls ever-exposed to asbestos were included. A dose-response relationship was observed in both groups but it was stronger in group 2. In comparison with subjects exposed at the minimum value of the cumulative index of exposure, the odds ratio was 2.6 (95\% CI 1.9-3.4) for subjects exposed to 75 fibres $\cdot \mathrm{mL}^{-1} \cdot \mathrm{year}^{-1}$ in group 1 increasing to 12.4 (95\% CI 4.6-33.7) in group 2 .

Our results suggest that the pleural carcinogenic effect of occupational asbestos exposure may be modified by additional exposure to RCF.

@ERSpublications

Increased pleural mesothelioma risk due to asbestos and refractory ceramic fibre co-exposure compared to asbestos http://ow.ly/yAx2D

Received: April 302014 | Accepted after revision: June 152014 | First published online: July 172014

Support statement: We received funding from ANSES - French Agency for Food, Environmental and Occupational Health and Safety (grant number: EST-2007-32).

Conflict of interest: None declared.

Copyright @ERS 2014 


\section{Introduction}

Refractory ceramic fibres (RCF) are manufactured inorganic fibres that belong to the subgroup man-made vitreous fibres. In 2002, the International Agency for Research on Cancer concluded that the limited available data from epidemiological studies did not allow adequate evaluation of the cancer risk linked with RCF exposure, thus, RCF were classified into group 2B: "possibly carcinogenic to humans" [1].

From animal studies, it has been shown that inhalation of RCF produced fibrosis and pleural tumours [2]. However, the experiment protocols were criticised and it has been hypothesised that a lung overload effect occurred in these studies [3].

From epidemiological studies, a US cohort study of workers exposed to RCF did not report any excess mortality related to all deaths, all cancers, or respiratory disease including mesothelioma [4]. However, the mean latency period in this cohort was only 21 years and only 942 subjects were available for analyses. In 2012, WALKER et al. [5] used the updated data of this cohort to compare the observed mesothelioma rates to those expected if RCF had the same carcinogenic potential as different varieties of asbestos. The authors rejected the hypothesis that RCF has the same pleural mesothelioma potency as crocidolite, but they could not reject the hypothesis for amosite or chrysotile $[5,6]$.

Besides cancer, the European and the US cohort studies of workers exposed to RCF revealed that the prevalence of pleural abnormalities increased with RCF exposure and in the absence of asbestos exposure [7-10]. More recently, a dose-response pattern was observed between cumulative exposure to RCF and radiographic pleural changes in workers not exposed to asbestos [11].

Animal models have shown that inflammation and fibrosis may be a preliminary step in the development of pleural mesothelioma [12]. Moreover, while controversial [13], there is some evidence from epidemiological studies of an increased risk of pleural mesothelioma in the presence of pleural plaques [14]. Since RCF seems to have the ability to induce pleural plaques in humans, we may hypothesise that RCF may also have the potential to induce a carcinogenic effect in the pleura.

In the case of RCF, the number of exposed workers is very small. Thus, it is not possible to perform cohort studies large enough to observe an increased risk of pleural mesothelioma due to such exposure. However, to date, there is no case-control study investigating the relationship between occupational RCF exposure and pleural mesothelioma. In France, a recent study examined the relationship between pleural mesothelioma and occupational co-exposure to asbestos and mineral wool. While it was almost impossible to reach a conclusion on the association of occupational exposure to mineral wool and pleural mesothelioma due to a low prevalence of exposure to mineral wool alone, without any occupational asbestos exposure, results from this study were in favour of an increased risk of pleural mesothelioma for subjects exposed to both asbestos and mineral wool compared to subjects only exposed to asbestos [15].

Using a French pooled case-control dataset, the objective of the present study was to investigate the hypothesis of increased pleural mesothelioma risk due to asbestos and RCF co-exposure compared with asbestos exposure alone.

\section{Methods}

Study design

The case-control dataset used in this study has been described previously [15, 16]. Cases were either newly diagnosed subjects included in a previous case-control study between 1987-1993 [17], or all histologically confirmed incident cases of pleural mesothelioma identified through the French National Mesothelioma Surveillance Program (Programme national de surveillance du mesothelioma; PNSM) from 1998 to 2006 [18]. Two controls were frequency matched to cases according to sex and year of birth within groups of 5 years from two population-based samples from the French Institute for Public Health Surveillance, which recorded complete job histories [19]. Finally, the present case-control dataset comprised 1199 male cases and 2379 male controls.

\section{Data collection}

A different, but standardised, questionnaire was administered to both cases and controls by trained interviewers. For each subject, age at diagnosis/interview, year of birth and complete job history were recorded. A job was defined as an occupation held within an industry. Occupations and industries were coded according to the International Standard Classification of Occupations [20] and International Standard Industrial Classification [21]. 
TABLE 1 Definition of exposure indicators included in the asbestos and refractory ceramic fibres (RCF) job exposure matrices used to assess occupational exposure of subjects

\begin{tabular}{|c|c|c|}
\hline Exposure indicators & Asbestos & RCF \\
\hline \multicolumn{3}{|l|}{ Probability \% } \\
\hline \multirow[t]{5}{*}{ Proportion of exposed workers within a job } & Possible (>0-5) & Class $1(>0.1-1)$ \\
\hline & Probable (>5-30) & Class $2(>1-10)$ \\
\hline & Likely (>30-70) & Class 3 (>10-50) \\
\hline & Definite (>70) & Class 4 (>50-90) \\
\hline & & Class $5(>90)$ \\
\hline \multicolumn{3}{|l|}{ Frequency \% } \\
\hline \multirow[t]{4}{*}{ Average frequency of exposure per working week } & Sporadic (>0-5) & Occasional (>0-5) \\
\hline & Occasional (>5-30) & Intermittent (>5-30) \\
\hline & Frequent $(>30-70)$ & Frequent $(>30-70)$ \\
\hline & Continuous (>70) & Permanent (>70) \\
\hline \multicolumn{3}{|l|}{ Intensity fibres $\cdot \mathrm{mL}^{-1}$} \\
\hline \multirow[t]{5}{*}{ Average level of exposure } & Very low $(>0-0.01)$ & Class $1(>0-0.1)$ \\
\hline & Low (>0.01-0.1) & Class $2(>0.1-1)$ \\
\hline & Medium (>0.1-1) & Class 3 (>1-3) \\
\hline & High $(>1-10)$ & Class $4(>3)$ \\
\hline & Very high $(>10)$ & \\
\hline
\end{tabular}

\section{Occupational exposure assessment}

Occupational asbestos and RCF exposures were assessed by two job exposure matrices (JEMs) provided by the French Institute for Public Health Surveillance [16, 22]. For each job classified in the international classifications and for different time periods, the JEMs provided three exposure indicators: 1) probability, 2) frequency, and 3) intensity of exposure (table 1). In order to be more specific, we defined a job exposed to asbestos as a job with a probability of exposure $>5 \%$ and a job exposed to RCF as a job with a probability of exposure $>1 \%$.

Next, subjects exposed to asbestos and RCF were defined as subjects with at least one exposed job (as defined previously). For each fibre type, three exposure indicators were defined: 1) the highest probability of exposure, 2) the total duration of exposure in years and, 3) the cumulative index of exposure expressed in fibres $\cdot \mathrm{mL}^{-1} \cdot$ year $^{-1}$. To calculate the cumulative index of exposure for both asbestos and RCF, we attributed values to each class of each semi-quantitative parameters included in both matrices. These values were arbitrarily defined by the centre of the class.

\section{Statistical analyses}

Because our objective was to study the quantitative effect of asbestos exposure in the presence or absence of RCF co-exposure on the risk of pleural mesothelioma, all our analyses were restricted to subjects occupationally exposed to asbestos (988 male cases and 1125 male controls).

All analyses were performed using unconditional logistic regression adjusted for age at diagnosis for cases and at interview for controls (years) and birth year. The occupational exposure variable of interest was the cumulative index of exposure to asbestos. To avoid imposing any functional form of dose-response relationship between cumulative index of exposure to asbestos, age and birth year variables and the logit of pleural mesothelioma, we used restricted cubic spline functions [23]. For each adjustment variable (age and birth year), we defined a priori three interior knots located at the 5th, 50th and 95th percentiles to ensure enough flexibility without producing a possible over fitting [23]. For the cumulative index of exposure to asbestos, the number of knots was defined by minimising the Akaike's information criterion from the model including all subjects occupationally exposed to asbestos. The model, including a four-knot restricted cubic spline located at the 25th, 50th, 75th and 95th percentiles, was retained.

To study the quantitative effect of asbestos exposure in the presence of RCF co-exposure on the risk of pleural mesothelioma, we did not include an interaction term between asbestos exposure and RCF exposure as there were no cases exposed to RCF that were not exposed to asbestos. Instead, we defined two groups of subjects: 1) those exposed to asbestos only, and 2) those exposed to both asbestos and RCF. Then, we estimated the dose-response relationship between occupational asbestos exposure and pleural mesothelioma in these two groups and compared them in terms of odds ratio and form of the dose-response relationship. 
As we used a cut-off of probability of exposure for both asbestos and RCF to define an exposed job, we performed several sensitivity analyses to assess the impact of this choice on our main results. Three additional scenarios were used to define a job exposed to asbestos or RCF: 1) any job with a probability of exposure $>0 \%$ for both asbestos and RCF; 2 ) any job with a probability of exposure $>30 \%$ for asbestos and $>1 \%$ for RCF; and 3) any job with a probability of exposure $>70 \%$ for asbestos and $>1 \%$ for RCF. All analyses were performed using SAS version 9.3 (SAS Institute Inc., Cary, NC, USA).

\section{Results}

The study sample included 988 male cases and 1125 male controls occupationally exposed to asbestos. As expected, most cases and controls were blue collar workers (69.9\% of cases and $65.2 \%$ of controls) (table 2).

Table 3 reports occupational asbestos exposure parameters according to occupational exposure to RCF (group 1 not exposed versus group 2 exposed). Among cases occupationally exposed to asbestos, 127 cases $(12.8 \%)$ were also exposed to RCF whereas $92(8.2 \%)$ controls were occupationally exposed to both asbestos and RCF. In group 2, 54.3\% of cases had definite occupational asbestos exposure versus $37.4 \%$ of cases in group 1. Cases exposed to both asbestos and RCF also had a greater cumulative index of exposure and a longer duration of exposure compared to cases not exposed to RCF.

Subjects exposed to both asbestos and RCF had a significantly higher risk of pleural mesothelioma compared to subjects only exposed to asbestos (OR 1.6, 95\% CI 1.2-2.2). However, while nonstatistically significant, the mean \pm SD average cumulative index of exposure for asbestos was higher in group 2 $\left(23.9 \pm 39.6\right.$ fibres $\cdot \mathrm{mL}^{-1} \cdot$ year $\left.^{-1}\right)$ compared to group $1\left(19.5 \pm 33.1\right.$ fibres $\cdot \mathrm{mL}^{-1} \cdot$ year $\left.^{-1}\right)$.

\section{TABLE 2 Characteristics of subjects occupationally exposed to asbestos}

Cases

Subjects $\mathbf{n}$
Age years
$<35$
$35-44$
$45-54$
$55-64$
$65-74$
$75-84$
$\geqslant 85$
Mean \pm SD (range)
Year of birth
$<1915$
$1915-1919$
$1920-1924$
$1925-1929$
$1930-1934$
$1935-1939$
$1940-1944$
$1945-1949$
$1950-1954$
$\geqslant 1955$

\section{Last occupation held}

0/1 Professional, technical and related worker

2 Administrative and managerial workers

3 Clerical and related workers

4 Sales workers

5 Service workers

6 Agricultural, animal husbandry, and forestry workers, fishermen and hunters

7/8/9 Production and related workers, transport, equipment operators and labourers

\section{Controls}

\begin{tabular}{cc}
988 & 1125 \\
$2(0.2)$ & $0(0.0)$ \\
$21(2.1)$ & $1(0.1)$ \\
$84(8.5)$ & $36(3.2)$ \\
$273(27.6)$ & $538(47.8)$ \\
$380(38.5)$ & $467(41.5)$ \\
$196(19.9)$ & $82(7.3)$ \\
$32(3.2)$ & $1(0.1)$ \\
$66.9 \pm 10.1(25-93)$ & $65.6 \pm 6.2(41-86)$ \\
& \\
$56(5.6)$ & $51(4.5)$ \\
$42(4.2)$ & $33(2.9)$ \\
$123(12.5)$ & $137(12.2)$ \\
$213(21.6)$ & $259(23.0)$ \\
$208(21.1)$ & $304(27.0)$ \\
$164(16.6)$ & $184(16.4)$ \\
$87(8.8)$ & $76(6.8)$ \\
$54(5.5)$ & $42(3.7)$ \\
$31(3.1)$ & $28(2.5)$ \\
$10(1.0)$ & $11(1.0)$ \\
& \\
$81(8.2)$ & $121(10.8)$ \\
$33(3.3)$ & $45(4.0)$ \\
$60(6.1)$ & $85(7.6)$ \\
$48(4.9)$ & $54(4.8)$ \\
$46(4.7)$ & $70(6.2)$ \\
$29(2.9)$ & $16(1.4)$ \\
$691(69.9)$ & $734(65.2)$ \\
& \\
\hline & \\
& \\
&
\end{tabular}

Data are presented as $\mathrm{n}(\%)$, unless otherwise stated. ${ }^{\#}$ : defined according to the seven major groups of the 1968 International Standard Classification of Occupations [21]. 
TABLE 3 Description of occupational asbestos parameters in subjects exposed to asbestos only and subjects exposed to both asbestos and refractory ceramic fibres (RCF)

\begin{tabular}{|c|c|c|c|c|}
\hline \multirow[t]{2}{*}{ Asbestos exposure parameters } & \multicolumn{2}{|c|}{ Exposed to asbestos only } & \multicolumn{2}{|c|}{ Exposed to asbestos and RCF } \\
\hline & Cases & Controls & Cases & Controls \\
\hline Subjects $\mathrm{n}$ & 861 & 1033 & 127 & 92 \\
\hline \multicolumn{5}{|l|}{ Highest probability of exposure } \\
\hline Probable & $151(17.5)$ & $320(31.0)$ & $0(0.0)$ & $6(6.5)$ \\
\hline Likely & $388(45.1)$ & $543(52.5)$ & $58(45.7)$ & $60(65.2)$ \\
\hline Definite & $322(37.4)$ & $170(16.5)$ & 69 (54.3) & $26(28.3)$ \\
\hline \multicolumn{5}{|l|}{ Total duration of exposure years } \\
\hline $0-10$ & $129(15.0)$ & $222(21.5)$ & $1(0.8)$ & $2(2.2)$ \\
\hline $10-20$ & $93(10.8)$ & $156(15.1)$ & $7(5.5)$ & $1(1.1)$ \\
\hline$>20$ & $639(74.2)$ & $655(63.4)$ & $119(93.7)$ & $89(96.7)$ \\
\hline \multicolumn{5}{|l|}{$\begin{array}{l}\text { Cumulative index of exposure } \\
\text { fibres } \cdot \mathrm{mL}^{-1} \cdot \text { year }^{-1}\end{array}$} \\
\hline $0-0.1$ & $107(12.4)$ & $204(19.7)$ & $0(0.0)$ & $2(2.2)$ \\
\hline $0.1-1$ & $162(18.8)$ & $252(24.4)$ & $10(7.9)$ & $21(22.8)$ \\
\hline $1-10$ & $203(23.6)$ & $274(26.5)$ & $55(43.3)$ & $53(57.6)$ \\
\hline $10-20$ & $91(10.6)$ & 77 (7.5) & $11(8.7)$ & $4(4.4)$ \\
\hline$>20$ & $298(34.6)$ & 226 (21.9) & $51(40.1)$ & 12 (13.0) \\
\hline
\end{tabular}

Data are presented as $\mathrm{n}(\%)$, unless otherwise stated.

Figure 1 represents the nonlinear effect of cumulative index of exposure to asbestos on the logit of pleural mesothelioma in subjects only exposed to asbestos (fig. 1a) and in subjects exposed to both asbestos and RCF (fig. 1b). The curve of both relationships was different, as was the magnitude of the effect. In group 1, the risk of pleural mesothelioma increased sharply up to $\sim 16$ fibres $\cdot \mathrm{mL}^{-1} \cdot$ year $^{-1}$ and then increased with a weaker slope thereafter. In group 2, the risk of pleural mesothelioma increased sharply to $\sim 22 \mathrm{fibres} \cdot \mathrm{mL}^{-1} \cdot \mathrm{year}^{-1}$ and was stable thereafter.

Table 4 reports some specific odds ratios for the association between pleural mesothelioma and asbestos cumulative index of exposure in group 1 and group 2 derived from the same model as in figure 1. A doseresponse relationship was observed in both groups but it seemed to be stronger in group 2 where subjects were exposed to both asbestos and RCF. Indeed, in group 1, the OR was 1.1 (95\% CI 1.0-1.1) for subjects exposed to 1 fibre $\cdot \mathrm{mL}^{-1} \cdot$ year $^{-1}$ compared to those exposed to the minimal cumulative index of exposure, and increased to 2.6 (95\% CI 1.9-3.4) for subjects exposed to 75 fibres $\cdot \mathrm{mL}^{-1} \cdot$ year $^{-1}$. In group 2 , the OR was 1.2 (95\% CI 1.1-1.4) for subjects exposed to 1 fibre $\cdot \mathrm{mL}^{-1} \cdot \mathrm{year}^{-1}$ compared to those exposed at the minimal cumulative index of exposure, and increased to 12.4 (95\% CI 4.6-33.7) for those exposed to 75 fibres $\cdot \mathrm{mL}^{-1} \cdot$ year $^{-1}$.

Sensitivity analyses presented similar results. Whatever the scenario considered, the curves of the doseresponse relationships in both group 1 and 2 were similar to those presented in figure 1 . Only the magnitude of the effect tended to be lower as the exposure assessment tended to be more specific but with the same difference between both groups (data not shown).

\section{Discussion}

Our results suggest that for the same cumulative index of exposure to asbestos, subjects exposed to both asbestos and RCF had a greater risk of pleural mesothelioma compared to subjects only exposed to asbestos. Indeed, in subjects who were only exposed to asbestos (group 1), the risk of pleural mesothelioma increased sharply up to 16 fibres $\cdot \mathrm{mL}^{-1} \cdot$ year $^{-1}$ and the magnitude of the effect was equivalent to an exposure of $\sim 4$ fibres $\cdot \mathrm{mL}^{-1} \cdot$ year ${ }^{-1}$ in the group of subjects exposed to both asbestos and RCF (group 2). In group 1 , the estimated effect of asbestos exposure increased with a weaker slope. The magnitude of the effect for subjects exposed to 100 fibres $\cdot \mathrm{mL}^{-1} \cdot$ year $^{-1}$ in group 1 was equivalent to the magnitude of the effect for subjects exposed to 7 fibres $\cdot \mathrm{mL}^{-1} \cdot$ year $^{-1}$ in group 2 . However, the slope after the fourth knot (corresponding to 106.2 fibres $\cdot \mathrm{mL}^{-1} \cdot$ year $^{-1}$ ) is hardly interpretable since restricted cubic spline functions impose two constraints of linearity before the first knot and after the last knot [24]. Thus, the increasing effect observed after the fourth knot should be carefully interpreted. In the group of subjects exposed to both asbestos and RCF (group 2), the estimated effect of asbestos exposure increased sharply up to $\sim 22$ fibres $\cdot \mathrm{mL}^{-1} \cdot \mathrm{year}^{-1}$ and 
a)

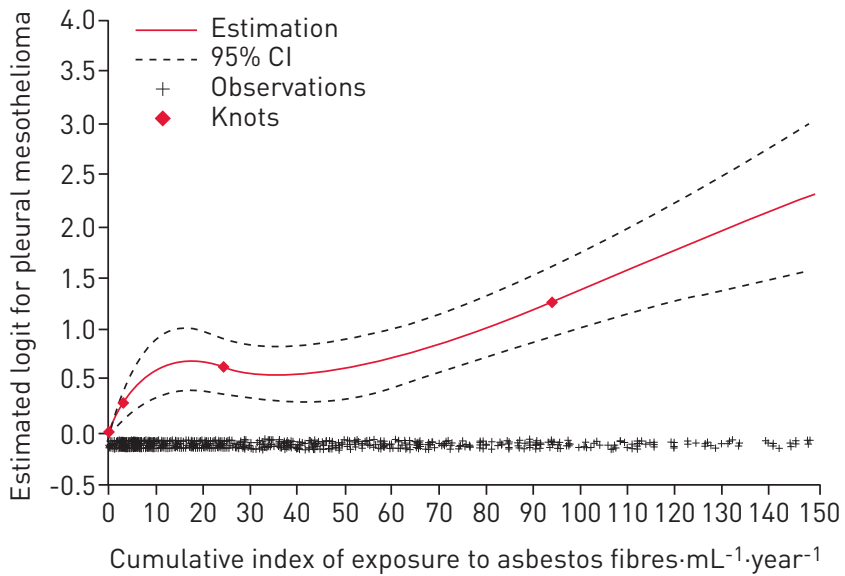

b)

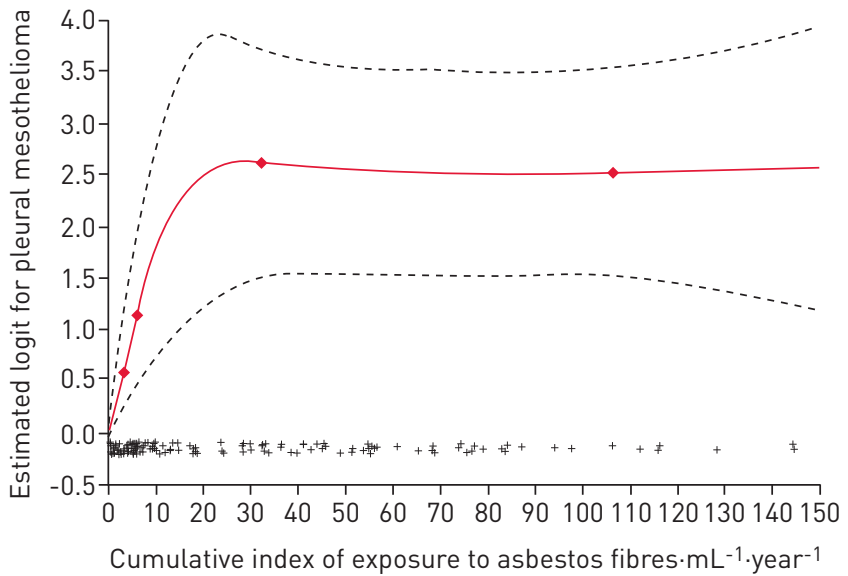

FIGURE 1 Nonlinear effect of cumulative index of exposure to asbestos on the logit of pleural mesothelioma in subjects a) exposed only to asbestos or b) exposed to both asbestos and refractory ceramic fibres relative to the minimum cumulative index of exposure. The solid line represents logistic estimates using a four-knot restricted cubic spline function located at the 25th, 50th, 75th and 95th percentiles of cumulative index of exposure to asbestos, adjusted for age and birth cohort using a three-knot restricted cubic spline functions located at the 5th, 50th and 95th percentiles. The odds ratio between two ever-exposed males with different cumulative index of exposure to asbestos values can be derived from the exponential of the difference between the y-axis values corresponding to the two specific values of cumulative index of exposure. Dashed lines are approximate $95 \%$ point-wise confidence intervals of the estimates compared with the reference value of cumulative index of exposure set at the minimum value of 0.0000875 .

was stable thereafter. However, confidence intervals were large and estimations were based on few subjects. Thus, the last part of the curve in group 2 should also be interpreted carefully.

As only two cases and three controls were not exposed to both asbestos and RCF simultaneously, we were unable to assess the effect of the sequence of exposure to asbestos and RCF in group 2.

There are two major hypotheses to explain the greater effect of asbestos exposure in subjects co-exposed to RCF: 1) an additional exposure to RCF may modify the carcinogenic response of asbestos by modifying intrapleural retention of asbestos fibres [25]; or 2) the effect of occupational exposure to RCF may be added to the effect of occupational asbestos exposure, implying that occupational RCF exposure has its own effect on pleural mesothelioma risk. Considering the first hypothesis, recently, a similar analysis performed on the same population study concluded that co-exposure to mineral wool and asbestos or co-exposure to crystalline silica and asbestos seemed to increase the risk of pleural mesothelioma [15]. To date, neither mineral wool nor crystalline silica are implicated in the aetiology of pleural mesothelioma, reinforcing the hypothesis that additional dust exposure (fibres or not) may interact with asbestos and modify the pleural carcinogenic response. Considering the second hypothesis, while animal studies are in favour of a carcinogenic effect of RCF [2], no epidemiological data are currently available on the carcinogenic potency of RCF [4-6, 26]. However, RCF are recognised to induce pleural plaques [7-9, 11], which are considered to

TABLE 4 Effect of the cumulative index of exposure to asbestos on pleural mesothelioma risk in subjects exposed to asbestos only and subjects exposed to both asbestos and refractory ceramic fibres (RCF)

\section{Cumulative index of exposure fibers $\cdot \mathrm{mL}^{-1} \cdot$ year $^{-1}$}

Exposed to asbestos only
Exposed to asbestos and RCF

\begin{tabular}{lcc}
\hline $\mathbf{0 . 0 0 0 0 8 7 5}$ & 1.0 & 1.0 \\
$\mathbf{0 . 1}$ & $1.0(1.0-1.0)$ & $1.0(1.0-1.0)$ \\
$\mathbf{1}$ & $1.1(1.0-1.1)$ & $1.2(1.1-1.4)$ \\
$\mathbf{1 0}$ & $1.9(1.4-2.5)$ & $5.9(2.1-16.4)$ \\
$\mathbf{2 5}$ & $1.9(1.5-2.4)$ & $13.7(4.0-46.7)$ \\
$\mathbf{5 0}$ & $1.9(1.4-2.5)$ & $12.8(4.7-35.1)$ \\
$\mathbf{7 5}$ & $2.6(1.9-3.4)$ & $12.4(4.6-33.7)$
\end{tabular}

Data are presented as OR $(95 \% \mathrm{Cl})$. The odds ratio was derived from a logistic regression model with cumulative index of exposure to asbestos (four-knot restricted cubic spline), age at diagnosis for cases and interview for controls (three-knot restricted cubic spline) and birth year (three-knot restricted cubic spline). 
be a marker of asbestos exposure [27]. Moreover, while controversial [2], there is some evidence that pleural plaques may be implicated in the development of pleural mesothelioma. Recently, results from a French cohort study of retired or unemployed workers previously occupationally exposed to asbestos showed a statistically significant association between pleural mesothelioma and pleural plaques while taking asbestos exposure into account (HR 6.8, 95\% CI 2.2-21.4) [14]. Finally, a recent review of animal studies raised the hypothesis that accumulation of asbestos fibres or carbon nanotubes in pleural spaces may led to pleural fibrosis and then mesothelioma [12]. Thus, the effect of RCF exposure on the risk of pleural mesothelioma seems biologically plausible and should be further investigated in humans.

It should be acknowledged that we did not have any information about the type of RCF used. Since the term RCF comprises different types of molecules, we may hypothesise that some RCF subgroups might have different oncogenic potential. Thus, the mixture of RCF types may have attenuated the dose-response relationship estimated in group 2. However, we do not believe that such mixtures have changed our general conclusion.

The main strength of this study is the sample size, which allowed us to reach a satisfactory statistical power for hypothesis testing. However, in any case, this sample size allowed us to reduce the asbestos exposure estimation uncertainty. Since both cases and controls came from different sources (a previous case-control study conducted in 1987-1993 [17] and the PNSM in 1998-2006 [18] for cases, and two samples recording complete job histories for controls) some potential biases could have been induced. Nevertheless, it has previously been shown that cases selected in the previous case-control study were representative of all French cases [17], and cases recorded in the PNSM were all incident cases identified in some specific, but representative, districts of France [18]. Thus, we believe that our pool of cases should be representative of all French cases of pleural mesothelioma during these two time periods. To identify any differences between cases included from the previous case-control study in 1987-1993 (cases 1) and cases from the PNSM in 1998-2006 (cases 2), we re-analysed the dataset by splitting the two sets of cases. For group 1 (subjects only exposed to asbestos), results between cases 1 and 2 were similar to those presented, while there were a slight difference in group 2. Whatever the set of cases, we still observed an increased risk of pleural mesothelioma in subjects exposed to both asbestos and RCF (group 2) compared to subjects only exposed to asbestos (group 1) and the form of the dose-response relationship was similar to that presented. However, the magnitude of the effect was higher when analysing data with cases 2 compared to cases 1 . Indeed, in group 2, cases 2 seemed to be exposed to asbestos at a higher level than cases 1 . However, due to the small number of cases 1 in group $2(n=36)$, and the large 95\% confidence intervals, the numeric difference is hardly interpretable and we should only retain that whatever the set of cases, the difference between group 1 and group 2 still persist (data not shown).

Controls were randomly selected from a sample that included the complete job history of 10010 subjects representative of the French population aged 25-74 years in 2007, and from a second sample made of 8344 controls from 15 population-based case-control studies. The representativeness of the first sample has been previously investigated and it has been shown that asbestos exposure was similar in subjects from this sample compared to the general French population [15]. The representativeness of the second sample has been published previously and no major distortion occurred with respect to the overall French male population in terms of occupational asbestos exposure for various time periods [19]. Overall, we believe that our subject selection did not induce major bias in the estimates of the effect of occupational asbestos exposure in the presence of occupational RCF exposure [28].

Occupational asbestos and RCF exposures were estimated with JEMs, which are recognised to be a good alternative to retrospective exposure assessment made by experts [29]. Indeed, JEMs were built by industrial hygienists and their assessments were based on available occupational measurement and job descriptions from epidemiological studies performed in France, as well as economic and statistical data. For a given homogeneous occupational group, the cells of the job exposure matrices carried an estimate of the probability, frequency and intensity of exposure. However, JEMs are known to produce misclassification that is likely to be non-differential in the context of case-control studies. Indeed, JEMs produce a standardised occupational exposure assessment for a given job without taking into account any intravariability or subject characteristics. Thus, some odds ratios might have been underestimated [29, 30]. Moreover, occupational exposure assessment made by a JEM relies on the reliability of job histories obtained from interviews and on the quality of coding. In all our data sources, trained interviewers obtained work history from the subjects, and previous studies found good validity and reliability of job histories obtained from interviews [31-36]. Moreover, there is no evidence that the recall of occupations is influenced by disease status $[31,32]$. A French study evaluated the quality of coding of job episodes collected by self-questionnaires among retired French males and found that the coding was satisfactory [37]. Finally, since results from sensitivity analysis were similar whatever the "ever exposure" definition, we are confident in our results and no major information bias should have occurred in these analyses. 
Since asbestos exposure was assessed by a JEM, we did not have any information on the types of asbestos used. However, in France, almost all the asbestos used was imported, and chrysotile seems to have been the most common type used. Sources of asbestos exposure mainly came from the construction industry, shipbuilding and the repairing industry, manufacture of asbestos cement products, metal working and motor vehicle manufacture or repair [17]. Thus, we did not expect subjects in group 1 to be exposed to a different type of asbestos compared with subjects in group 2.

It is well known that arbitrary categorisation of a quantitative variable, such the asbestos cumulative index of exposure, avoids imposing any specific functional form of the covariate-outcome relationship but also induces a loss of information and reduction in power [38, 39]. More importantly, imposing some arbitrary categories may not help identify the true dose-response relationship or differences between two doseresponse relationships. However, it is not better to introduce a continuous variable in regression models that assume a specific mathematical relationship between exposure and disease when this assumption is violated. In the case of logistic regression, the relationship between the continuous variable and the log odds of the disease has to be linear which is not accurate in the case of occupational asbestos exposure and pleural mesothelioma [40]. One alternative is to use restricted cubic spline functions, which allow the estimation of smooth and plausible dose-response curves [23,38]. One disadvantage of restricted cubic spline functions is that the form of the dose-response relationship may depend on the location and the number of knots. Thus, we used the recommendation of DESQUILBET and MARIOTTI [23] for both adjustment variables and exposure variables in order to obtain a more accurate dose-response curve.

In conclusion, according to our results in this large case-control study, the dose-response relationship between occupational asbestos exposure and pleural mesothelioma is stronger in the presence of occupational co-exposure to RCF. Along with previously published experimental and epidemiological data, these results should be regarded as a new element of the discussion concerning potential pleural effect of RCF with or without asbestos co-exposure. Further investigations are needed to confirm and understand the mechanisms of the effect modification of asbestos exposure in the presence of co-exposure to RCF.

\section{Acknowledgements}

We would like to thank Yuriko Iwatsubo (Département Santé Travail, Institute de Veille Sanitaire, Saint Maurice, France) for allowing us to use data collected from the previous mesothelioma case-control study. We would also like to thank the members of PNSM technical committee who are not listed as authors (Philippe Astoul (Service d'Oncologie Thoracique, Maladie de la Plèvre et Pneumologie Interventionnelle, Hopital Nord, Aix-Marseille Universite, Marseille, France), Soizick Chammings (Institut Interuniversitaire de Medecine du Travail de Paris Ile de France, Crèteil, France), Daniele Luce (Departement Sante Travail, Institut de Veille Sanitaire), Catherine Frenay (Service d'Oncologie Thoracique, Maladie de la Plèvre et Pneumologie Interventionnelle, Hopital Nord, Aix-Marseille Universite), Françoise Galateau-Salle (Multicentrique a Vocation Nationale des Mesotheliomes Pleuraux (MESONAT), Hopital de la Cote de Nacre, Caen, France), Nolwenn Le Stang (Multicentrique a Vocation Nationale des Mesotheliomes Pleuraux (MESONAT), Hopital de la Cote de Nacre)) for allowing us to use data collected from the PNSM, and Ray Cook (Département Langues et Culture, Université Bordeaux, Bordeaux, France) for his careful reading of this manuscript.

\section{References}

1 World Health Organization, International Agency for Research on Cancer. IARC Monographs on the Evaluation of Carcinogenic Risks to Humans. Vol. 81. Man-made Vitreous Fibres. Lyon, IARC, 2002.

2 Utell MJ, Maxim LD. Refractory ceramic fiber (RCF) toxicity and epidemiology: a review. Inhal Toxicol 2010; 22 : 500-521.

3 Brown RC, Bellmann B, Muhle H, et al. Survey of the biological effects of refractory ceramic fibres: overload and its possible consequences. Ann Occup Hyg 2005; 49: 295-307.

4 LeMasters GK, Lockey JE, Yiin JH, et al. Mortality of workers occupationally exposed to refractory ceramic fibers. J Occup Environ Med 2003; 45: 440-450.

5 Walker AM, Maxim LD, Utell M. Risk analysis for mortality from respiratory tumors in a cohort of refractory ceramic fiber workers. Regul Toxicol Pharmacol 2002; 35: 95-104.

6 Walker AM, Maxim LD, Utell MJ. Corrigendum. Are airborne refractory ceramic fibers similar to asbestos in their carcinogenicity? Inhal Toxicol 2012; 24: 928-929.

Cowie HA, Wild P, Beck J, et al. An epidemiological study of the respiratory health of workers in the European refractory ceramic fibre industry. Occup Environ Med 2001; 58: 800-810.

8 Lentz TJ, Rice CH, Succop PA, et al. Pulmonary deposition modeling with airborne fiber exposure data: a study of workers manufacturing refractory ceramic fibers. Appl Occup Environ Hyg 2003; 18: 278-288.

9 Lockey JE, LeMasters GK, Levin L, et al. A longitudinal study of chest radiographic changes of workers in the refractory ceramic fiber industry. Chest 2002; 121: 2044-2051.

10 Lockey J, Lemasters G, Rice C, et al. Refractory ceramic fiber exposure and pleural plaques. Am J Respir Crit Care Med 1996; 154: 1405-1410.

11 Lockey JE, Roggli VL, Hilbert TJ, et al. Biopersistence of refractory ceramic fiber in human lung tissue and a 20-year follow-up of radiographic pleural changes in workers. J Occup Environ Med 2012; 54: 781-788.

12 Donaldson K, Murphy FA, Duffin R, et al. Asbestos, carbon nanotubes and the pleural mesothelium: a review of the hypothesis regarding the role of long fibre retention in the parietal pleura, inflammation and mesothelioma. Part Fibre Toxicol 2010; 7: 5. 
13 Goldberg M. Are lung and pleural benign asbestos induced diseases a preliminary step in the pathogenic process of mesothelioma and lung cancer development? Occup Environ Med 2005; 62: 663-664.

14 Pairon JC, Laurent F, Rinaldo M, et al. Pleural plaques and the risk of pleural mesothelioma. J Natl Cancer Inst 2013; 105: 293-301.

15 Lacourt A, Gramond C, Audignon S, et al. Pleural mesothelioma and occupational co-exposure to asbestos, mineral wool and silica. Am J Respir Crit Care Med 2013; 187: 977-982.

16 Lacourt A, Leffondre K, Gramond C, et al. Temporal patterns of occupational asbestos exposure and risk of pleural mesothelioma. Eur Respir J 2012; 39: 1304-1312.

17 Iwatsubo Y, Pairon JC, Boutin C, et al. Pleural mesothelioma: dose-response relation at low levels of asbestos exposure in a French population-based case-control study. Am J Epidemiol 1998; 148: 133-142.

18 Goldberg M, Imbernon E, Rolland P, et al. The French National Mesothelioma Surveillance Program. Occup Environ Med 2006; 63: 390-395.

19 Goldberg M, Banaei A, Goldberg S, et al. Past occupational exposure to asbestos among men in France. Scand J Work Environ Health 2000; 26: 52-61.

20 International Labour Office. International Standard Classification of Occupations. Revised Edition 1968. Geneva, International Labour Organization, 1968.

21 United Nations. International Standard Industrial Classification of All Economic Activities. Rev. 2. New York, United Nations; 1968.

22 Fevotte J, Dananche B, Delabre L, et al. Matgene: a program to develop job-exposure matrices in the general population in France. Ann Occup Hyg 2011; 55: 865-878.

23 Desquilbet L, Mariotti F. Dose-response analyses using restricted cubic spline functions in public health research. Stat Med 2010; 29: 1037-1057.

24 Durrleman S, Simon R. Flexible regression models with cubic splines. Stat Med 1989; 8: 551-561.

25 Davis JM, Jones AD, Miller BG. Experimental studies in rats on the effects of asbestos inhalation coupled with the inhalation of titanium dioxide or quartz. Int J Exp Pathol 1991; 72: 501-525.

26 Walker AM, Maxim LD, Utell MJ. Are airborne refractory ceramic fibers similar to asbestos in their carcinogenicity? Inhal Toxicol 2012; 24: 416-424.

27 Clarke CC, Mowat FS, Kelsh MA, et al. Pleural plaques: a review of diagnostic issues and possible nonasbestos factors. Arch Environ Occup Health 2006; 61: 183-192.

28 Lacourt A. Reply: No indication that mineral wool causes mesothelioma. Am J Respir Crit Care Med 2013; 188: 873-874.

29 Bouyer J, Dardenne J, Hemon D. Performance of odds ratios obtained with a job-exposure matrix and individual exposure assessment with special reference to misclassification errors. Scand J Work Environ Health 1995; 21: 265-271.

30 Kauppinen TP, Mutanen PO, Seitsamo JT. Magnitude of misclassification bias when using a job-exposure matrix. Scand J Work Environ Health 1992; 18: 105-112.

31 Baumgarten M, Siemiatycki J, Gibbs GW. Validity of work histories obtained by interview for epidemiologic purposes. Am J Epidemiol 1983; 118: 583-591.

32 Bond GG, Bodner KM, Sobel W, et al. Validation of work histories obtained from interviews. Am J Epidemiol 1988; 128: $343-351$.

33 Bourbonnais R, Meyer F, Theriault G. Validity of self reported work history. Br I Ind Med 1988; 45: 29-32.

34 Eskenazi B, Pearson K. Validation of a self-administered questionnaire for assessing occupational and environmental exposures of pregnant women. Am J Epidemiol 1988; 128: 1117-1129.

35 Pershagen G, Axelson O. A validation of questionnaire information on occupational exposure and smoking. Scand J Work Environ Health 1982; 8: 24-28.

36 Stewart WF, Tonascia JA, Matanoski GM. The validity of questionnaire-reported work history in live respondents. J Occup Med 1987; 29: 795-800.

37 Pilorget C, Imbernon E, Goldberg M, et al. Evaluation of the quality of coding of job episodes collected by self questionnaires among French retired men for use in a job-exposure matrix. Occup Environ Med 2003; 60: 438-443.

38 Greenland S. Dose-response and trend analysis in epidemiology: alternatives to categorical analysis. Epidemiology 1995; 6: 356-365.

39 Zhao LP, Kolonel LN. Efficiency loss from categorizing quantitative exposures into qualitative exposures in casecontrol studies. Am J Epidemiol 1992; 136: 464-474.

40 Hodgson JT, Darnton A. The quantitative risks of mesothelioma and lung cancer in relation to asbestos exposure. Ann Occup Hyg 2000; 44: 565-601. 